\title{
Effect of Heating Rate and Annealing Temperature on Secondary Recrystallization of Goss Grains in a Grain Orientated Silicon Steel
}

\author{
Fatayalkadri CITRAWATI, ${ }^{1,3)}$ Md Zakaria QUADIR ${ }^{1,2)}$ and Paul Richard MUNROE ${ }^{1 / *}$ \\ 1) School of Materials Science and Engineering, University of New South Wales, Kensington, NSW 2052 Australia. \\ 2) Microscopy \& Microanalysis Facility, John de Laeter Centre, Curtin University, Bentley, WA 6102 Australia. \\ 3) Research Center for Metallurgy and Materials, Indonesian Institute of Sciences, Tangsel, 15314 Indonesia.
}

(Received on December 12, 2016; accepted on March 7, 2017)

\begin{abstract}
In this investigation, a grain oriented silicon steel was annealed from a starting state of its primary recrystallized condition by varying time, temperature and heating rate to elucidate the phenomena that occur during the secondary recrystallization growth of Goss grains. Goss secondary recrystallization occurs in a temperature range from 900 to $1000^{\circ} \mathrm{C}$. Using a slow rate of heating $\left(5^{\circ} \mathrm{C} /\right.$ minute) to the annealing temperature yields more scattering in the Goss orientation during the early stages of secondary growth, compared with rapid heating to the annealing temperature in a preheated furnace. Irrespective of the early stage annealing events, after extended annealing to 300 minutes, Goss texture dominates through both rapid and slow heating annealing. Goss growth starts from the sub-surface regions of the sheet, where the concentration of $\eta$-fibre grains is high. At a higher annealing temperature $\left(1000^{\circ} \mathrm{C}\right)$ faster Goss growth occurs at more frequent sites and, as a result, when the process is completed the average size of Goss grains are smaller than that observed through annealing at $900^{\circ} \mathrm{C}$. These investigations were conducted by annealing in an air-circulating furnace followed by extensive orientation measurements through electron backscattered diffraction (EBSD) in a field emission gun scanning electron microscope (FEG SEM) and analysis using a transmission electron microscope (TEM).
\end{abstract}

KEY WORDS: secondary recrystallization; grain growth; annealing; Goss; silicon steels.

\section{Introduction}

The process of electrical power transmission from power generation stations to residential, industrial and commercial areas, involves the use of grain oriented silicon steels as a soft magnetic material in transformer cores. Compared to some other magnetic materials these alloys are not only economic, but also yield a high magnetic flux under an applied field of $800 \mathrm{~A} / \mathrm{m}\left(\mathrm{B}_{800}\right) .{ }^{1-3)}$ These properties are related to the presence of Goss grains in the alloy, which have a crystallographic orientation having the $<100>$ direction parallel to the rolling direction (RD) and $\{110\}$ plane parallel to the rolling plane (ND) of the steel sheets. These are then arranged in laminates in the transformer core. It has been demonstrated that $\langle 100\rangle$ is the easiest magnetization direction for a bec lattice. ${ }^{4,5)}$ Therefore having a high density of Goss grains serves to increase the magnetic anisotropy of the steel through altering the shape of the hysteresis loop, and hence, reduces hysteresis losses. However, in industrially processed polycrystalline steel sheets grains are often deviated from the ideal Goss $\{110\}<100>$ orientation. In a given grain the angular distance from the ideal Goss orientation is called the deviation angle, which has been used to describe the sharpness of the Goss texture in the material. ${ }^{6-8)}$

\footnotetext{
* Corresponding author: E-mail: p.munroe@unsw.edu.au DOI: http://dx.doi.org/10.2355/isijinternational.ISIJINT-2016-718
}

The formation of Goss texture in the steel is generated through complex thermomechanical processing starting from the melt to the final recrystallization anneal. ${ }^{9-11)}$ As a result, there are a larger number of process variables involved, although variables related to steel chemistry and downstream processing schedules have been thoroughly studied. ${ }^{12,13)}$ After obtaining an ingot with a particular chemical composition, the subsequent sheet processing involves a sequence of hot and cold rolling stages, together with recrystallization anneals. Based on the steel chemistry and cold rolling schedules grain oriented Si-steels are divided in two broad classes: one-pass or two-pass. ${ }^{2,14)}$ Those sheets that are produced from one pass cold rolling are also known as high permeability grade steels, ${ }^{15,16)}$ while the conventional grain oriented silicon steel (CGO) grades are produced from two-pass cold rolling. ${ }^{17,18)} \mathrm{A}$ major difference between these two grades is in the use of inhibitors in the melt: $\mathrm{MnS}$ and AlN particles form in the high permeability grade ${ }^{19)}$ but only $\mathrm{MnS}$ particles form in CGO. ${ }^{20)}$ During the final annealing process, certain Goss oriented grains grow significantly faster to produce very large grains compared to the matrix grains. Since this growth process is highly orientation selective it is known as secondary recrystallization or abnormal grain growth. ${ }^{21,22)}$

According to the literature, abnormal growth of Goss grains is facilitated by pinning and restriction of growth of the matrix grains. ${ }^{19,23-25)}$ The theories explaining abnormal 
growth fall into two broad categories: oriented nucleation and growth selectivity. ${ }^{26,27)}$ Between these two theories, the growth selectivity theory has been more broadly supported in the literature, since the first development of these alloys in 1934. This theory relies on the presence of coincidence site lattice (CSL) boundaries around the Goss grains and these types of boundaries are assumed to be less strongly pinned by inhibiting particles due to their early dissolution during annealing. This, thus, allows Goss grains to grow exclusively and consume the matrix grains. ${ }^{28,29)}$ The other theory, which is based on orientation nucleation, is derived from the notion that Goss grains are larger in the primary recrystallization state and therefore during secondary growth they grow preferentially. ${ }^{30,31)}$ As noted above, this theory is, however, relatively less well supported in the literature.

In spite of extensive research, the exact mechanisms that drive exclusive Goss growth amongst other oriented grains remains unresolved. ${ }^{28,29,32-34)}$ It is interesting to note that although Goss texture sharpness is a vitally important property, only limited research has been done to investigate this phenomenon. ${ }^{35)}$ It is noted that the CSL theory largely relies on the sharpness of Goss grains in the sense that a perfect Goss orientation is more likely to be surrounded by grains having a CSL relationship and, therefore, has a growth advantage over other orientations. Following this notion Ushigami, et al. ${ }^{17,36)}$ found that the sharp Goss grains in the primary recrystallized structures grow earlier at lower temperatures compared to the more strongly deviated Goss grains. Therefore, Goss sharpness seems to be strongly affected by the secondary recrystallization temperature. ${ }^{36,37)}$ In addition to this, Sokolov, et al. ${ }^{37)}$ observed that a sharp Goss texture forms within a small window of annealing temperature (typically from $860^{\circ} \mathrm{C}$ to $1050^{\circ} \mathrm{C}$ ), outside of which the orientation deviation from the ideal Goss increases dramatically. In their study the details of material chemistry and processing schedule (whether this is single cold rolled or double cold rolled) were not noted.

The differences in material chemistry and processing schedule between the CGO and high permeability grades have implications on the secondary grain growth mechanisms and textures. In this study the effects of annealing conditions, on the secondary grain growth in a CGO grade primary recrystallized sheet is elucidated by conducting a series of annealing experiments at varying temperatures, times and heating rates. The structural and orientation changes in this study are investigated by scanning electron microscopy (SEM), electron backscattered diffraction (EBSD) and transmission electron microscopy (TEM).

\section{Experimental}

In this study, a commercially produced CGO silicon steel was studied. This was heat treated to the primary recrystallized condition and exhibited a distribution of equiaxed grains $15 \pm 10 \mu \mathrm{m}$ in diameter. The chemical composition of the as-received material was evaluated using inductively coupled plasma - optical emission spectrometry (ICP-OES) and the data are presented in Table $\mathbf{1}$. The presence of Mn and $\mathrm{S}$ suggests the existence of $\mathrm{MnS}$ particles as inhibitors to grain growth. Briefly, on the primary recrystallized sheet processing, the hot-band was cold rolled in two steps, in the
Table 1. Chemical composition of as received Fe-Si alloy.

\begin{tabular}{cccccccccc}
\hline Element & $\mathrm{Cr}$ & $\mathrm{Ni}$ & $\mathrm{Mn}$ & $\mathrm{Mo}$ & $\mathrm{S}$ & $\mathrm{P}$ & $\mathrm{Si}$ & $\mathrm{Cu}$ & $\mathrm{Fe}$ \\
\hline$\% \mathrm{wt}$ & 0.095 & 0.09 & 0.06 & 0.01 & 0.020 & 0.005 & 3.5 & 0.2 & 95.3 \\
\hline
\end{tabular}

Table 2. Annealing parameters.

\begin{tabular}{rcc}
\hline $\mathrm{T}\left({ }^{\circ} \mathrm{C}\right)$ & $\mathrm{t}(\mathrm{min})$ & Heating Rate $\left({ }^{\circ} \mathrm{C} / \mathrm{min}\right)$ \\
\hline 900 & $5,10,30,60,300$ & $\mathrm{RH}$ \\
1000 & $5,10,30,60,300$ & $\mathrm{RH}$ \\
900 & $5,10,30,60,300$ & $\mathrm{SH} @ 5^{\circ} \mathrm{C} / \mathrm{min}$ \\
\hline
\end{tabular}

first step the sheet was rolled to $0.7 \mathrm{~mm}$ thickness, it was then subjected to an annealing treatment at $950^{\circ} \mathrm{C}$ in a $\mathrm{H}_{2} /$ $\mathrm{N}_{2}$ gas atmosphere, and then in the second step the sheet was further cold rolled to $0.30 \mathrm{~mm}$ thickness. Finally, the sheet was subjected to a decarburization annealing treatment in $\mathrm{H}_{2} / \mathrm{N}_{2}$ atmosphere for $4-5$ minutes at $820-830^{\circ} \mathrm{C}$.

For conducting heat treatments, samples with dimensions of $30 \mathrm{~mm} \times 4 \mathrm{~mm}$ were subjected to annealing treatments at $800^{\circ} \mathrm{C}$ or $1050^{\circ} \mathrm{C}$ for times ranging from 5 to 300 minutes in one of two heating schedules (rapid heating $(\mathrm{RH})$ and slow heating $(\mathrm{SH})$ ). In the $\mathrm{RH}$ schedule the samples were placed into the furnace chamber after the furnace temperature had reached and stabilized at the target temperature. In the SH schedule the samples were placed inside the furnace chamber at room temperature and then the temperature was ramped up at $5^{\circ} \mathrm{C} / \mathrm{min}$ rate. Annealing was carried out in an air-circulating furnace at $\pm 5^{\circ}$ variations once the annealing temperature was reached. Following annealing, all samples were air cooled outside the furnace chamber. The parameters used in the annealing experiments are listed in Table 2.

An electron back scattered diffraction (EBSD) investigation of the section perpendicular to the transverse-direction (TD) and normal-direction (ND) was conducted after preparing the surfaces by mechanical polishing with colloidal silica. EBSD data acquisition was performed using an Oxford Instruments system attached in a Carl Zeiss Auriga Dual Beam scanning electron microscope (SEM). During EBSD scans a $20 \mathrm{kV}$ beam was rastered with 1 to $3.5 \mu \mathrm{m}$ step size over a predetermined area. The EBSD data analysis was conducted using HKL Channel $5^{\mathrm{TM}}$ software. Specimens for transmission electron microscopy (TEM) were prepared by mechanical thinning to $\sim 100 \mu \mathrm{m}$ followed by twin jet eletropolishng using a Tenupol 5 machine that operates at $35 \mathrm{~V}$ applied DC potential using a 10:90 volume ratio of perchloric acid (70\%) and methanol chemical mixture. Polished specimens were examined in a Philips CM200 TEM operating at $200 \mathrm{kV}$ to which energy dispersive spectrosocpy (EDS) facilities were interfaced.

\section{Results}

The EBSD measured orientation maps and corresponding $<200>$ pole figures of the as-received and 5 minute RH annealed at $900^{\circ} \mathrm{C}$ samples are shown in Figs. 1 and 2. Figure 1(a) is a small representative area of a large scan covering an area $\sim 4 \mathrm{~mm}$ in length along the $\mathrm{RD}$ and full sheet thickness. In this map the grains within a $15^{\circ}$ angular distance from the Goss, $\eta$ - orientations and $\gamma$-orientations 


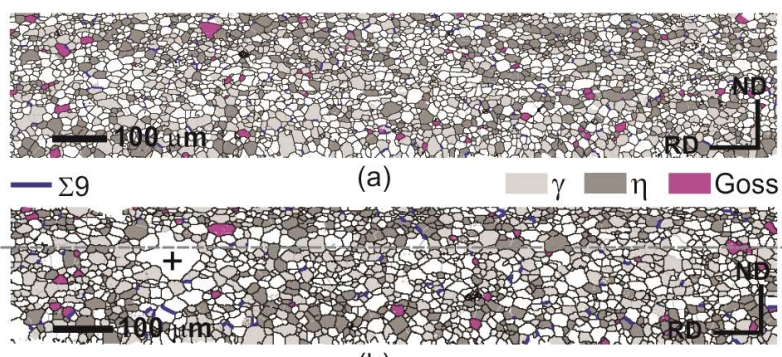

(b)

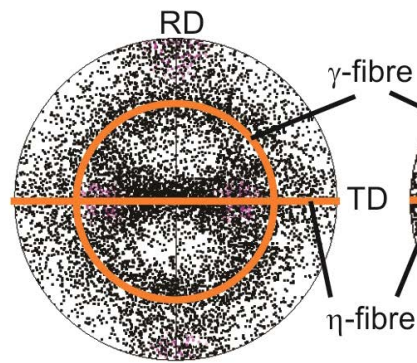

(c)

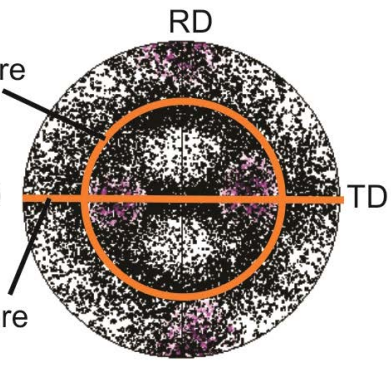

(d)

Fig. 1. EBSD measured orientation maps of the RD-ND crosssection of (a) as received and (b) 5 minute RH annealed (at $900^{\circ} \mathrm{C}$ ) samples showing the distributions of Goss, $\eta$-, and $\gamma$-fibre grains, and (c)-(d) their corresponding $<200>$ pole figures. (Online version in color.)

(a)

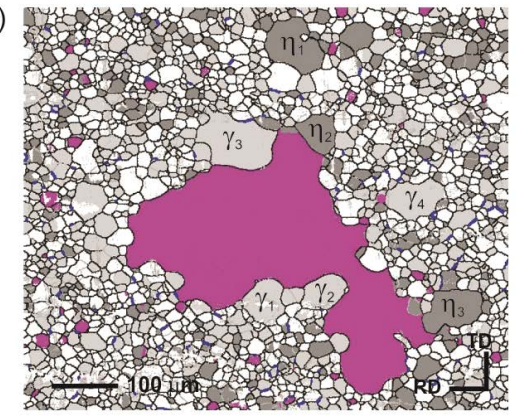

(b)

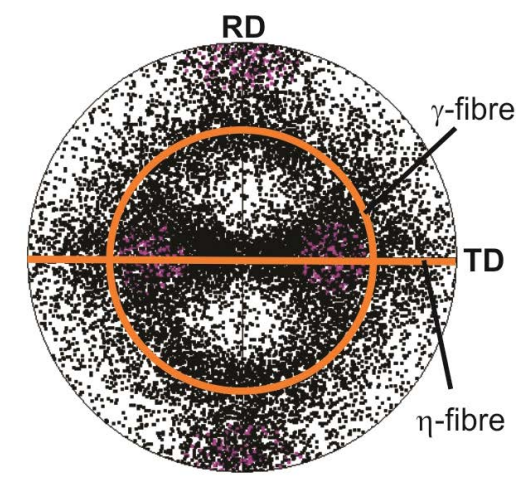

Fig. 2. (a) EBSD measured orientation map in the RD-TD crosssection showing a larger Goss grain growing into the ordinary grain growth matrix and (b) corresponding $<200>$ pole figure showing the overall orientations of the matrix grains in the sample RH annealed for 5 minutes at $900^{\circ} \mathrm{C}$. (Online version in color.)

are plotted in fuchsia (in online version), dark grey and light grey, respectively, and the other orientations are plotted in white. From a thorough examination from several EBSD scans the following key features of the Goss oriented grains are summarized:

- The size distribution of Goss oriented grains is identical to the overall grain distribution in the sample. The size of the largest Goss grains $(\sim 30 \mu \mathrm{m})$ is also comparable to the largest grains of other orientations, and this indicates that Goss grains do not have size advantages in the primary recrystallized state.

○ There is no preferential spatial distribution of Goss grains across the sheet thickness. Of note, the larger Goss grains $(>25 \mu \mathrm{m})$, which are of special focus since the exhibit a size advantage, are also found to be evenly distributed.

○ The coincident site lattice (CSL) $\Sigma 9$ boundaries, that have been thought to play a key role in Goss growth $\left.^{29,32-34,38)}\right)$, present only in a very small fraction of grains (see the thick blue grain boundary lines) and are nonpreferentially associated with a broad range of orientations of the primary recrystallized textures. The $\Sigma 9$ boundaries are plotted using Brandon's criteria ${ }^{39)}$ and only occupy $\sim 0.6 \%$ of the total boundary length over a $2-62.5^{\circ}$ misorientation range.

- The misorientation histograms of the grain boundaries enclosing the Goss grains are comparable with the overall boundary distributions in the sample. However, as shown in Fig. 1 when the Goss grains are surrounded by $\eta$-fibre grains the next neighbour histogram changes. This is shown in detail in Fig. 3, whereby the misorientation angle of the aggregate is distributed over a higher angular range than the Goss grains.

$\circ$ The orientation perfections of the Goss grains are not related to their size i.e. the larger Goss grains $(>25 \mu \mathrm{m})$ do have closer orientations to ideal Goss locations.

The $<200>$ pole figure in Fig. 1(c) is plotted from a large scan from which Fig. 1(a) was cropped. In this pole figure one orientation data per grain is plotted. Overall, the orientations are distributed along two major fibres: the $\gamma$ - and $\eta$-fibres, which are marked by the superimposed gray (orange in online version) lines. The grains in the $\gamma$-fibre have $<111>$ parallel to the ND and the grains in the $\eta$-fibre have $<100>$ parallel to the RD of the sheet. The Goss orientation is located in the centre of the $\eta$-fibre. In terms of the spatial distribution of the $\gamma$ - and $\eta$-fibre grains, the $\gamma$-oriented grains are evenly distributed without having an obvious location preference, but the $\eta$ grains are located in the subsurface region (see the colour coded plots in Fig. 1(a)).

Annealing experiments performed with the $\mathrm{RH}$ schedule were conducted over a temperature range from $800^{\circ} \mathrm{C}$ to $1050^{\circ} \mathrm{C}$. No secondary grain growth occurs below $900^{\circ} \mathrm{C}$ and therefore data from annealing at $900^{\circ} \mathrm{C}$ (as the lowest temperature considered here) is presented. After 5 minutes of annealing at $900^{\circ} \mathrm{C}$, overall grain growth occurred (see the orientation map in Fig. 1(b)). Remarkably, the grains in the subsurface regions (along the dotted line) have achieved greater growth relative to elsewhere in this section. In this map, the $\eta$ - and $\gamma$-grains are colour-coded as dark grey and light grey, respectively, and thereby it is found that the $\eta$ and $\gamma$ grains are located near the subsurface and middle regions of the sheet, respectively. However, there are no Goss oriented grains within the grown grains. Notably, the largest observed grain (marked + in Fig. 1(b)) has a $\{102\}<251>$ orientation, which is at $35.4^{\circ}$ angular distance from the ideal Goss orientation. Since the grains in the subsurface regions achieve faster growth, the rolling plane sec- 
tion (the plane at right angle to the ND) at this thickness was thoroughly investigated, and, thus, evidence of an extended Goss growth was captured and shown in Fig. 2(a). There are other grown grains surrounding the giant Goss grain. Their sizes are similar to that found in the TD cross section $($ marked + ) in Fig. 1(b). These growing grains belong to the $\gamma$ - and $\eta$-fibres. Among the $\gamma$ grains the (111)[-23-1], (323) [03-2], (111)[2-1-1], (223)[03-2] orientations are marked and likewise among the $\eta$ grains the (001)[-100], (2 3 13) [8-1-1], (203)[1-60] orientations are marked. These $\gamma$ and $\eta$ grains exhibit faster growth than the overall matrix, but are still much smaller than the giant Goss grain shown in Fig. 2(a). It is noteworthy that there are many Goss grains in the matrix having orientations within $15^{\circ}$ spread from the ideal Goss, but they do not achieve abnormal growth. This indicates that the neighbouring grain orientation relationship of a Goss grain is important for promoting abnormal growth. This finding has been reported in many other studies. ${ }^{36,40-43)}$ Practically, once growth of a Goss grain has occurred, to some extent the neighbouring grains become consumed and
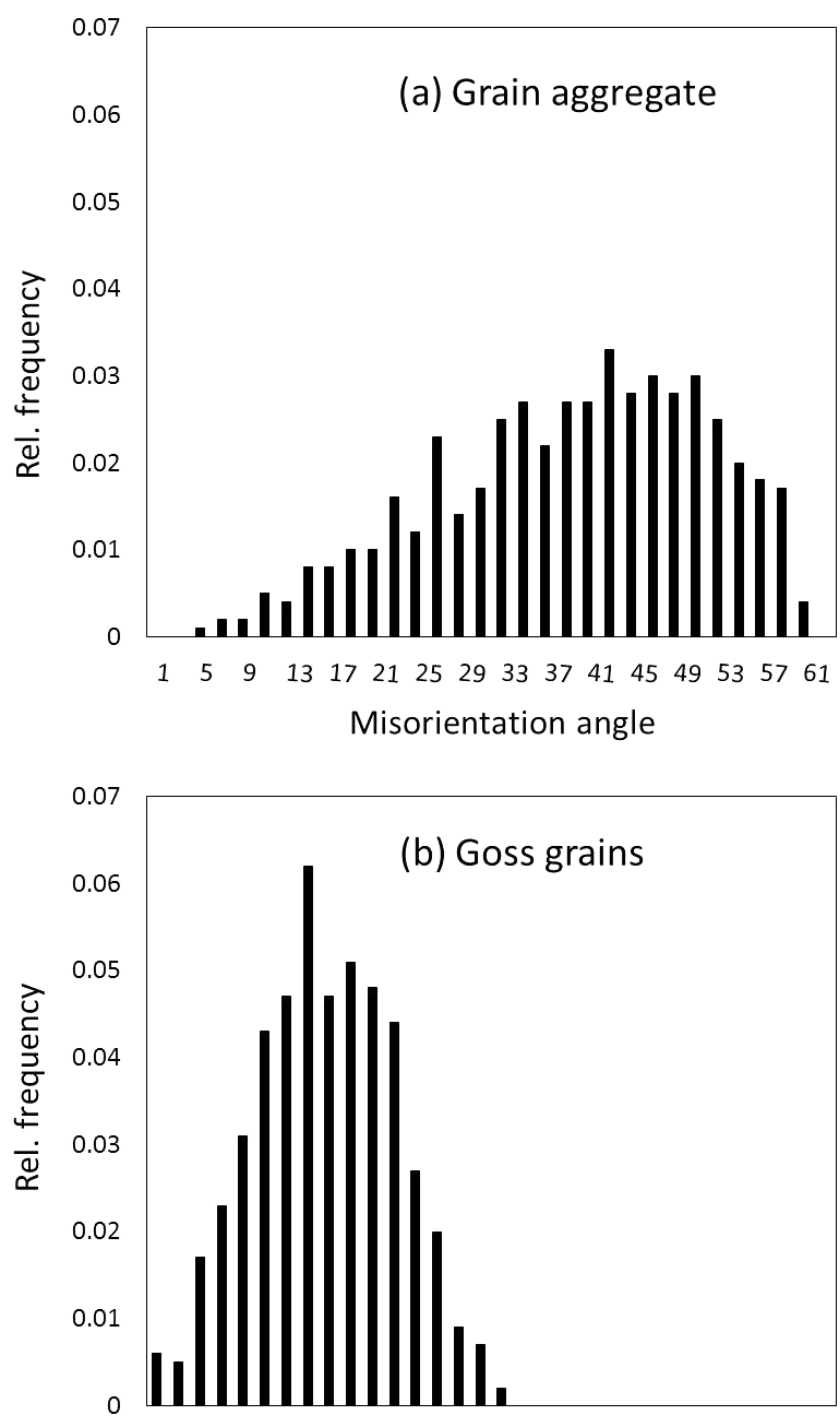

$\begin{array}{llllllllllllllll}1 & 5 & 9 & 13 & 17 & 21 & 25 & 29 & 33 & 37 & 41 & 45 & 49 & 53 & 57 & 61\end{array}$ Misorientation angle

Fig. 3. Misorientation angle distributions of the (a) whole grain aggregate and (b) Goss grains with neighboring $\eta$-fiber grains. their orientation information is lost. This has been one of the prime technical challenges that hinder addressing the central question of this topic i.e. how growth of Goss grains initiates at the very early stages of secondary recrystallization annealing. Overall, the texture of the matrix grains in the 5 minutes annealed sample remains identical to the primary recrystallized textures with predominance of the $\eta$ - and $\gamma$-fibres (compare Figs. 1(c) and 1(d) with 2(b)).

\subsection{Structural Developments during the Growth Pro- cess}

The progress of grain growth is observed as a function of heating rate, temperature and time. Figure $\mathbf{4}$ contains a series of representative orientation maps capturing structural developments under different annealing conditions. Each map in Fig. 4 represents a small area from a large number of scans for finding true representation of growth. Depending on the size of the growing grains in a given sample, the number of such scans can be as many as 42. Figures 4(a)4(c) show the nature of grain growth after 10 to 60 minutes of $\mathrm{RH}$ annealing at $900^{\circ} \mathrm{C}$. In Fig. 4(a), two growing grains are identified within the scan area: one is $22.1^{\circ}$ (white) and the other is $8.8^{\circ}$ (gray (pink in online version)) away from the ideal Goss orientation. The other regions show normal growth of matrix grains. In the 30 minutes annealed sample in Fig. 4(b) some grains are grown through the entire sample thickness. Then, during subsequent heating to 60 and then 300 minutes, secondary grain growth reaches completion through the entire sample sheet thickness (see Fig. 4(c)). In addition, there are some grains trapped near the mid-thickness of the sheet. These are typically known as island grains. Two features of island grains are of particular interest: (a)
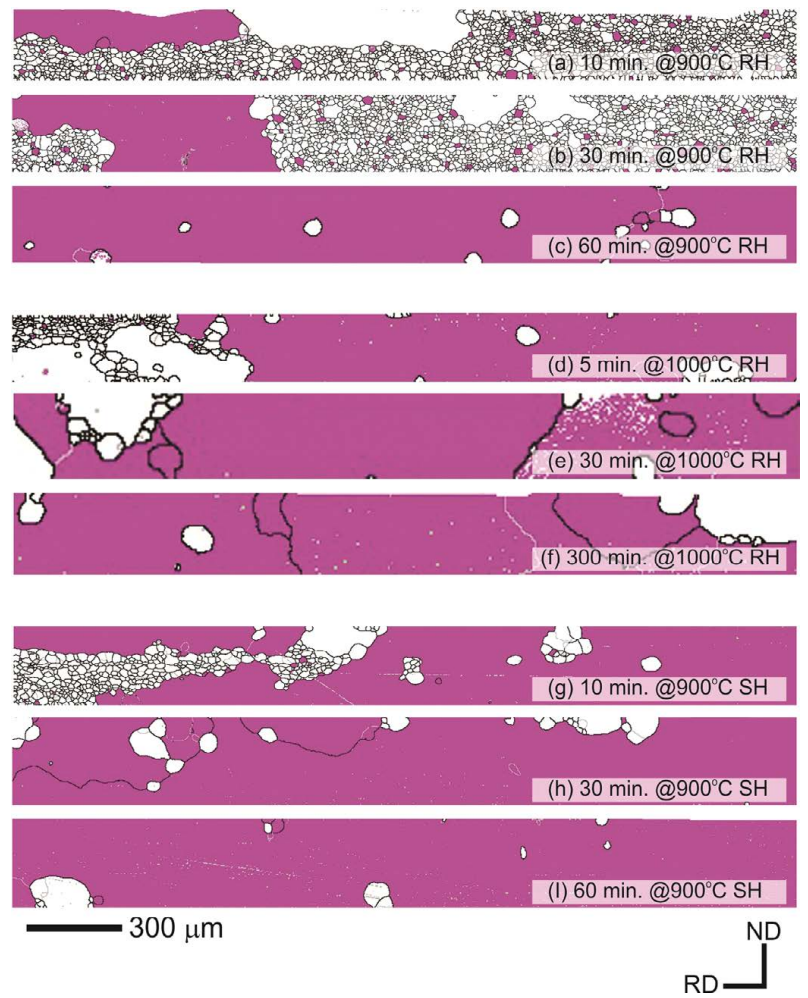

Fig. 4. EBSD measured orientation maps showing the growth of Goss grains during $\mathrm{RH}$ and $\mathrm{SH}$ annealing at $900^{\circ} \mathrm{C}$ and $1000^{\circ} \mathrm{C}$. (Online version in color.) 
despite extending the annealing time up to 300 minutes some island grains still remain present and (b) some island grains are lined up along the mid-thickness or at the surface of the sheet. The former feature, i.e. their sustainability, indicates that presumably some sort of local orientation relationships across their boundaries with the growing Goss grains renders these boundaries highly immobile.

Figures 4(d)-4(f) show orientation maps of sheets that were $\mathrm{RH}$ annealed at $1000^{\circ} \mathrm{C}$. Identical growth phenomena to that at $900^{\circ} \mathrm{C}$ were observed and at the end of the annealing treatment this resulted in very large secondary grains. Nevertheless, the following exceptions in their growth phenomena are also identified:

- Faster growth occurs in the earlier annealing stages: the grains after 5 minutes annealing at $1000^{\circ} \mathrm{C}$ are larger than the $900^{\circ} \mathrm{C}$ sample after 10 minutes (compare Figs. 4(d) with 4(a)).

- At the completion of secondary growth the grains in the $1000^{\circ} \mathrm{C}$ sample are smaller than those in $900^{\circ} \mathrm{C}$ sample (compare Figs. 4(f) with 4(c)).

$\circ$ The island grains are larger in the $1000^{\circ} \mathrm{C}$ annealed sample compared with those in the $900^{\circ} \mathrm{C}$ annealed sample. In either case they remain stable after extended annealing up to 300 minutes (Fig. 4(f))

In the samples that were annealed by slow heating $(\mathrm{SH})$ at $5^{\circ} \mathrm{C} / \mathrm{min}$ rate to $900^{\circ} \mathrm{C}$ the annealing time is measured once the temperature reached $900^{\circ} \mathrm{C}$ and, therefore, faster recrystallization was obtained compared with the RH samples (compare Figs. 4(g) with Fig. 4(a) and 4(d)). Moreover, the average size of secondary recrystallized grains in the $\mathrm{SH}$ annealed samples is larger than the RH samples (compare Figs. 4(g) with 4(a)). It should be noted that some differences in the grain sizes are not apparent in the EBSD maps presented in Fig. 4. For instance, grain size differences between Figs. 4(c) and 4(i) are not readily apparent, because the same field of view is maintained in all the images presented in Fig. 4 (see the single scale bar for all the panels). However, the difference becomes more pronounced when large area data was measured covering almost $12 \mathrm{~cm}$ along the sample length.

It has been found from extensive EBSD scans that the RH annealed samples at $900^{\circ} \mathrm{C}$ exhibit more island grains than the equivalent $\mathrm{SH}$ annealed samples. However, it is interesting to note that the spatial distribution of island grains following $\mathrm{RH}$ annealing at $900^{\circ} \mathrm{C}$ and $1000^{\circ} \mathrm{C}$ are different. In the $900^{\circ} \mathrm{C}$ annealed sample the island grains are largely present in the surface and mid-thickness regions and in the $1000^{\circ} \mathrm{C}$ annealed sample they are distributed randomly across the entire sample thickness. This presumably happens due to the differences in the growth chronology at these two annealing temperatures and will be discussed later.

\subsection{Texture Evolution}

According to the observations shown in Fig. 2(a) (rolling plane) secondary recrystallized grains are easily distinguishable through their size differences with matrix grains. In this

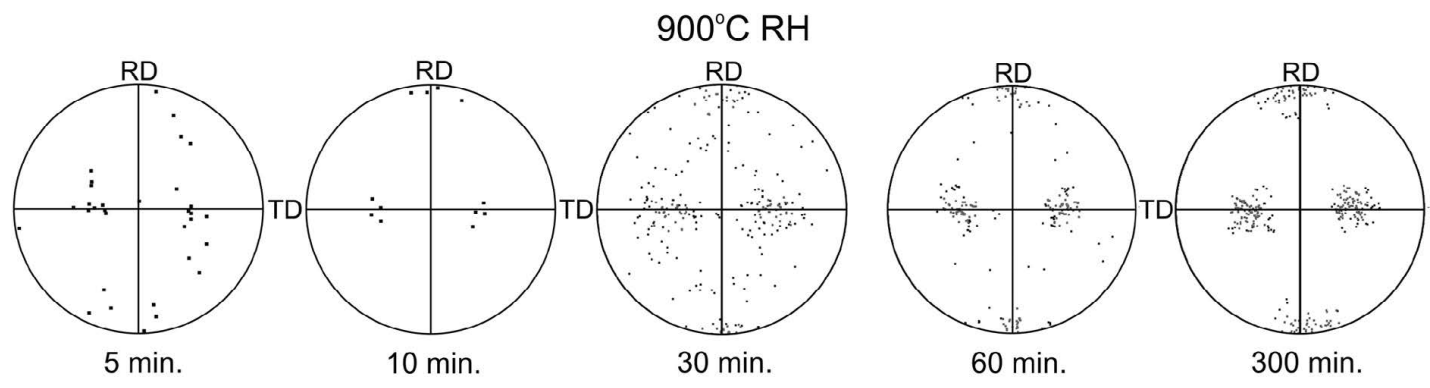

$1000^{\circ} \mathrm{C} \mathrm{RH}$
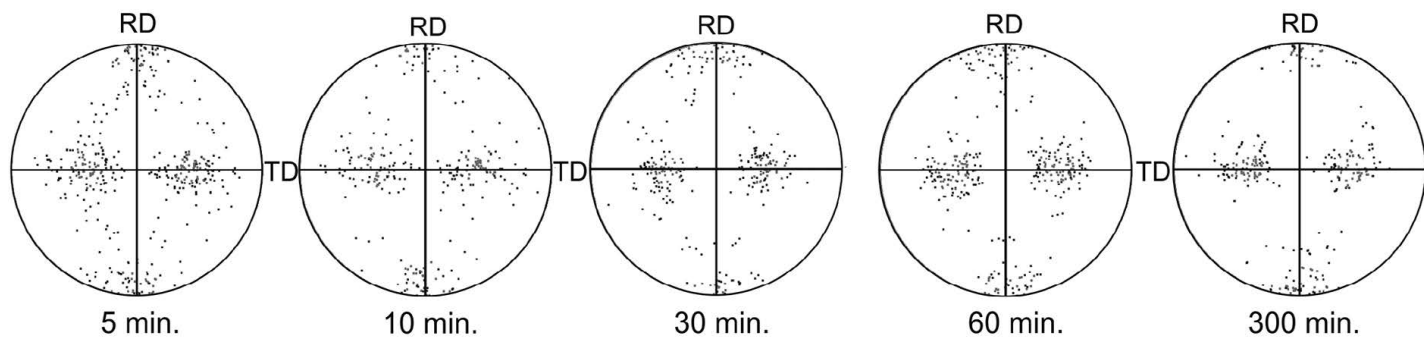

$900^{\circ} \mathrm{C} \mathrm{SH}$
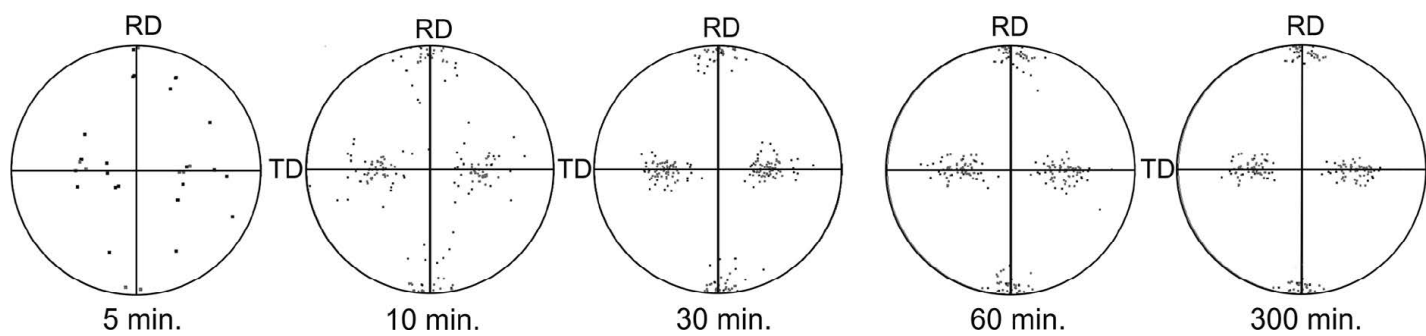

Fig. 5. A series of $<200>$ pole figures showing orientations of secondary Goss grains following RH and $\mathrm{SH}$ annealing at $900^{\circ} \mathrm{C}$ and $1000^{\circ} \mathrm{C}$ 
image the grains over $500 \mu \mathrm{m}$ in diameter are considered to have achieved secondary/abnormal growth. Although some other grains also have grown larger than the average matrix aggregate, their growth is limited below $100 \mu \mathrm{m}$ in diameter, and, therefore, are considered to have undergone conventional grain growth. Figure 5 shows a series of $<200>$ pole figures that represent the orientations of grains that achieved secondary growth during $\mathrm{RH}$ annealing at $900^{\circ} \mathrm{C}$ and $1000^{\circ} \mathrm{C}$ and $\mathrm{SH}$ annealing at $900^{\circ} \mathrm{C}$. In these pole figures one orientation point per grain is plotted. Only a small number of grains were found to achieve secondary growth after 5 and 10 minutes RH annealing at $900^{\circ} \mathrm{C}$. However, the number of secondary Goss grains increases with increasing annealing time. It should be noted, however, that annealing at $1050^{\circ} \mathrm{C}$ significantly deteriorates the formation of secondary Goss texture and, therefore, was not characterized further in this investigation. Identical findings were noted from an early investigation by May and Turnbull, showing the detrimental effects of high annealing temperatures $\left(1050^{\circ} \mathrm{C}\right.$ to $\left.1150^{\circ} \mathrm{C}\right)$ on both Goss texture and magnetic properties. ${ }^{44)}$

There are two key phenomena that were found as annealing progresses:

0 The Goss texture intensifies with annealing time as this is shown by outlining the orientation spread around the ideal Goss. The spread around the Goss orientations is symmetrical.

- The number of secondary recrystallized grains, other than those with Goss orientations, becomes fewer with increasing annealing time. This is apparent from the reduction in the scattered orientation points with annealing time. That is, compare the scattered points in the samples annealed for 30, 60 and 300 minutes at $900^{\circ} \mathrm{C}$ (in Fig. 5). This implies that although some non-Goss grains achieve secondary growth during the early annealing stages, later these grains become consumed by the growing Goss grains having orientations closer to the ideal Goss (within $15^{\circ}$ ).

In comparison to the samples annealed at $900^{\circ} \mathrm{C}$, the secondary growth at $1000^{\circ} \mathrm{C}$ show some differences in the sense that some $\eta$ - and $\gamma$ - orientated grains also achieve secondary growth during the early stages of annealing for 5 and 10 minutes. This is indicated from the oblong-shaped orientation spread of Goss, the spread is $\pm 25^{\circ}$ along the ND-TD line from the ideal Goss orientation. However, as the growth continues the $\eta$ - and $\gamma$ - oriented secondary grains become consumed by Goss grains and a symmetric orientation distribution around the Goss orientation is established. Unlike the RH annealing at 900 and $1000^{\circ} \mathrm{C}$ the grain growth in $\mathrm{SH}\left(5^{\circ} \mathrm{C} / \mathrm{min}\right)$ at $900^{\circ} \mathrm{C}$ is more orientation selective to Goss. This becomes apparent when the equivalent pole figures at $900^{\circ} \mathrm{C}$ RH with $900^{\circ} \mathrm{C} \mathrm{SH}$ in Fig. 5 are compared after extended annealing. However, in the earlier heating stages during the $\mathrm{SH}$ annealing (e.g. 5 minutes) the secondary grain orientations are almost random.

During the growth of the secondary Goss grains the orientations of the remaining matrix grains that experience some conventional growth were monitored and it was found that they maintain, overall, the same recrystallization texture as the as-received material (containing the $\eta$ - and $\gamma$-fibers). This trend remains unchanged up to the end of the secondary growth process in all the samples. To demonstrate

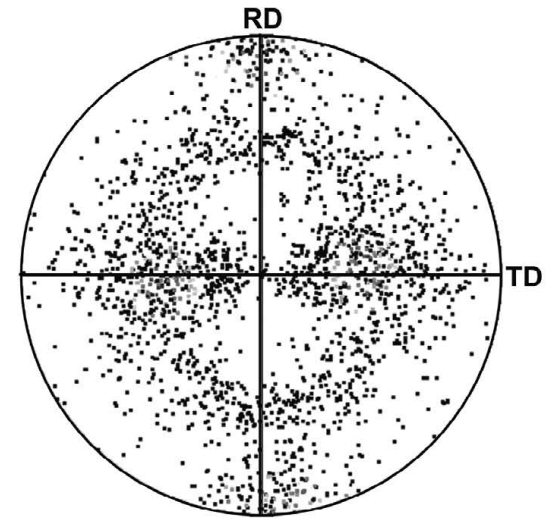

(a) Matrix grains

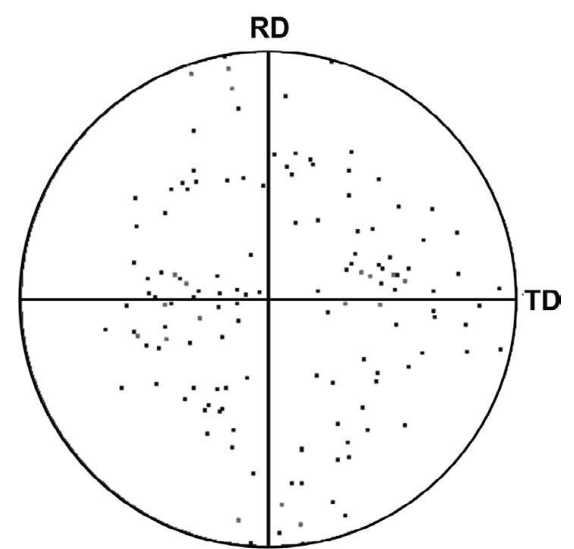

(b) Island grains (46 grains)

Fig. 6. EBSD measured $<200>$ pole figures showing orientations of (a) matrix grains and (b) island grains in the sample isothermally annealed for 60 minutes at $1000^{\circ} \mathrm{C}$.

this phenomenon a representative $<200>$ pole figure is presented in Fig. 6(a) from the 60 minutes annealed RH sample at $1000^{\circ} \mathrm{C}$. An identical texture distribution to that of the as received material is identified containing the $\eta$ - and $\gamma$-fibers (Fig. 1).

In all the annealing experiments performed, some island grains were found located in the interior of the large secondary recrystallized grains (see Fig. 4 for example). The three dimensional connectivity of island grains was often questioned in concern to sectioning artifacts. Fig. 6(b) shows that the orientation of the island grains in the sample annealed for 60 minutes at $1000^{\circ} \mathrm{C}$, which are identical to the primary recrystallization texture of the matrix grains. A general orientation check of the island grains in all the samples was conducted and no orientation preference with the grain enclosing them was found. This phenomenon was maintained in all the samples although the nature of island grains varied with annealing conditions, such that, island grains were larger and more commonly observed when annealing was conducted at higher temperatures (e.g. $\left.1000^{\circ} \mathrm{C}\right)$.

\section{Discussion}

In this investigation, the temperature range from 900 to $1000^{\circ} \mathrm{C}$ was found to be critically important for the abnormal growth of Goss grains. This was identified in two ways. Firstly, abnormal growth of Goss was not achieved 
when isothermal annealing was conducted outside this temperature range and, secondly, the early growing grains during the slow heating experiments were not Goss oriented (since it is highly likely that growth started below $900^{\circ} \mathrm{C}$ ). These observations indicate that the inhibiting particles play a critical role in the exclusive determination of Goss growth. Although no theory has been unanimously accepted for accounting for the Goss growth mechanism, the role of inhibitor particles has been generally regarded to be critical, since it has been found that without these inhibiting particles Goss growth does not occur. ${ }^{20)}$ A recent summary of all major theories of the growth of Goss grains and their limitations are described in an article by Morawiec. ${ }^{45)}$ Based on the chemistry of the current material (in Table 1) the most probable inhibiting particles are likely to be MnS. Figure 7(a) shows bright field TEM images of the as received primary recrystallized material, showing a dispersion of particles at both grain boundaries and in the grain interiors. The particle size varies in a range from 20-300 nm. TEM-based energy dispersive spectroscopy (EDS) analysis shows these particles are primarily composed of $\mathrm{Mn}$ and $\mathrm{S}$ (see the line scan profile across the particle show in Fig. 7(b). This particle is located at the boundary of two grains and thus resists the grain boundary movement, as shown in the STEM image in the inset of the EDS spectrum in Fig. 7(b). The effective-
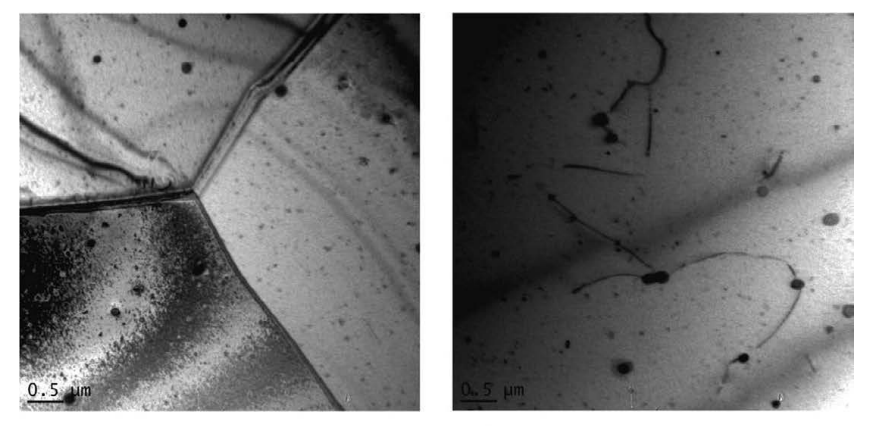

(a) BF TEM

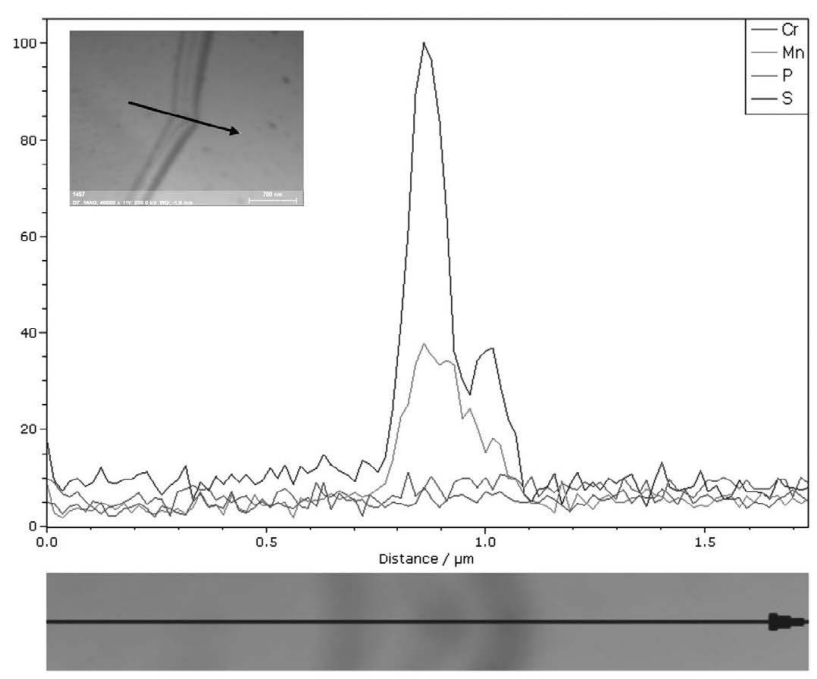

(b) EDS

Fig. 7. (a) TEM bright field images showing dispersion of particles in the grain boundaries and grain interiors together with a small number of residual dislocations and (b) EDS line scan analysis of a particle located at a grain boundary in the as received material. ness of the selective pinning is thought to be realized by the smaller, more coherent particles, however direct evidence is yet to be found. Further experiments based on dynamic annealing are required to investigate this further.

The primary role of these particles is to stabilise the matrix by making it resistant to growth and, thus, provide a growth advantage to certain grains, in this case Goss grains. This means that the more uniform the matrix, the less drag force is required to be exerted by the particles. ${ }^{27)}$ The matrix stability is not only important to instigate Goss growth during the early annealing stages, but also at all instances as annealing progresses, and thus Goss growth is assured to continue. Therefore, a chronological development in the microstructure is important to understand how (a) Goss grains initiate growing, (b) matrix grains remain homogeneous and (c) island grains form. Figure $\mathbf{8}$ shows several orientation maps that are captured at different growth conditions in a $900^{\circ} \mathrm{C} \mathrm{RH}$ annealed sample. In Fig. 8(a) the grains near the subsurface regions achieve more growth than those near the centre and, thus, the size homogeneity starts breaking up and giant Goss grains start growing in the subsurface regions. It was shown in Fig. 1 that the $\eta$-fibre texture dominates at the surface region and this indicates that Goss grains have some growth advantages if they are associated with $\eta$-fibre grains. Likewise, Goss grains may also grow from the other sides of the cross-section and, thus, the growing grains meet near the centreline (Figs. 8(b) and 8(c)). Meanwhile some grains closer to the centre also achieve primary growth and become larger than the matrix (in Fig. 8(b)). They become trapped between two growing Goss grains at the centre line. When they get trapped as a cluster they may agglomerate together to form one or two larger grains and appear as island grains. In the next stage of the growth process the secondary grown Goss grains from one subsurface region of the sheet cross-
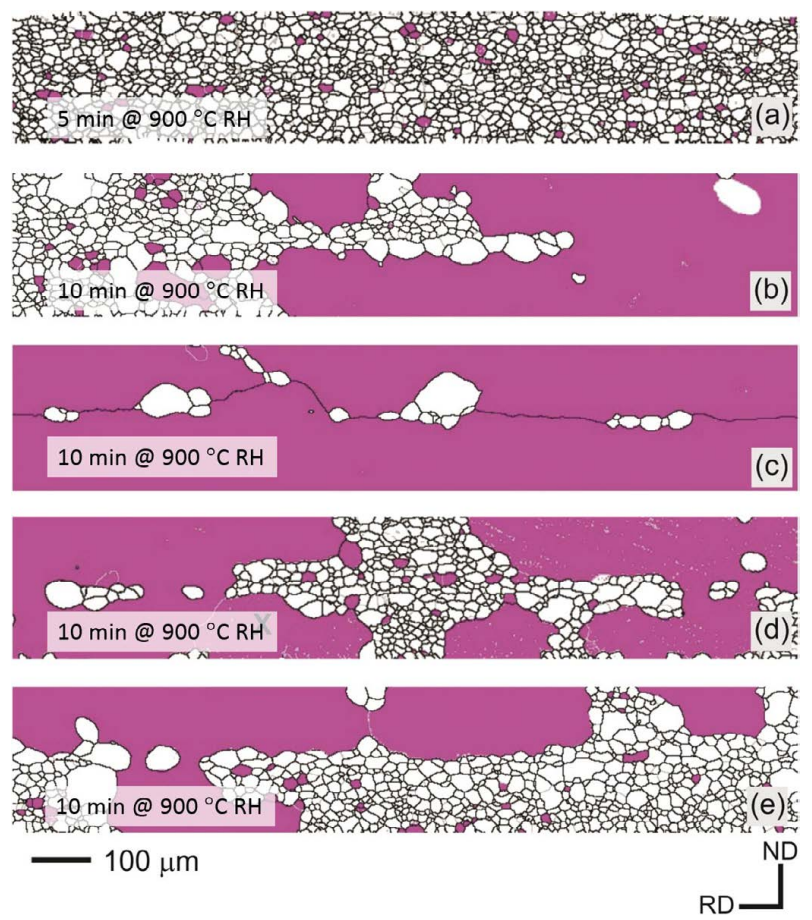

Fig. 8. A series of EBSD measured orientation maps capturing different conditions in $900^{\circ} \mathrm{C} \mathrm{RH}$ annealing for elucidating island grain formations inside Goss secondary grains. (Online version in color.) 
section invade into the secondary grown Goss grain that has formed on the other subsurface region of the sheet, but still cannot consume the island grains and, as a result, the island grains thus become arranged along the centre line of the sheet as shown in Fig. 4(c). An example of a Goss grain consuming another Goss grain after having undergone secondary growth is shown in Fig. 8(d), whereby the Goss grain formed in the top subsurface is in the process of consuming the Goss grain from the bottom subsurface (marked $\mathrm{X})$. In some situations secondary growth occurs from only one side of the sheet and then it grows to the other side. In this case the Goss grains grow around the large grains and, thus, entrap them as island grains (see Fig. 8(e)). In this case, the island grains may not essentially line up along the centre line. The growth morphology of the island grains, thus, provides a fingerprint of the growth chronology of the Goss grains in the matrix. For example, island grains in Fig. 4(c) in the $900^{\circ} \mathrm{C}$ annealed samples lined up along the centre line, but in Fig. 4(f) in the $1000^{\circ} \mathrm{C}$ annealed samples they are distributed in a more random pattern. It is interesting to note that there is no orientation preference of the island grains although they have highly immobile boundaries with the Goss grains (Fig. 6(b)).

In this investigation the variations in the nature of secondary grains growth with heating rate and annealing temperature are clear. These become apparent from the differences in secondary grain size, and which, in turn, is related to the frequency of secondary grain formation. In this research, EBSD mapping was conducted over a distance up to 15 $\mathrm{cm}$ in length along the $\mathrm{RD}$ to obtain a statistical validated result on the grain size measurements. The size of a grown grain was measured from the length along the $\mathrm{RD}$, since the growth along ND is restricted by the sheet thickness to 0.3 $\mathrm{mm}$. The data is presented in Fig. 9 as an average grain size against annealing time. In the $\mathrm{RH} 1000^{\circ} \mathrm{C}$ annealed samples the recrystallized grain size grew to a length of $\sim 1.0 \mathrm{~mm}$ long and then reached saturation. According to the growth chronology shown in Fig. 8, this indicates that secondary recrystallization takes place at distances apart of $\sim 1 \mathrm{~mm}$ and then these grains grow to consume the primary recrystallized matrix until encountering another growing secondary grain. Once two growing grains meet the growth process

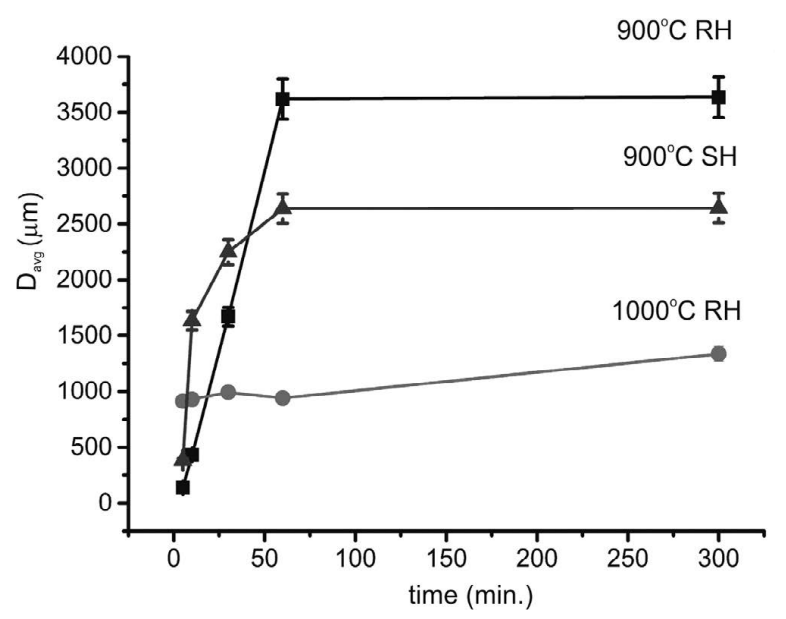

Fig. 9. A plot showing the variation of average size of secondary annealing grown grains with annealing time. slows down and reaches saturation. Therefore with further annealing up to 5 hours further growth becomes negligible. In the same mechanism, at $900^{\circ} \mathrm{C} \mathrm{RH}$ annealing the grains continue growing to $3.5 \mathrm{~mm}$ up to 60 minutes annealing and then saturation is achieved. Therefore at $900^{\circ} \mathrm{C}$ successful secondary grain growth occurs over $3.5 \mathrm{~mm}$ average distances and the final grain size is larger.

From the aforementioned observation, it is obvious that at a higher annealing temperature secondary growth of Goss occurs at more frequent locations, which is more likely due to faster dissolution/coarsening of the inhibiting particles at the higher temperature. It may also be considered that there is less restriction on boundary conditions at higher annealing temperatures that makes a specific Goss grain easier to grow. The corresponding $<200>$ pole figures shown in Fig. 5 demonstrate that there is a larger spread in the secondary grain orientations in the sample $\mathrm{RH}$ annealed at $1000^{\circ} \mathrm{C}$, which supports the concept of relaxation in orientation selectivity at higher temperatures. In the $\mathrm{SH}$ sample annealed at $900^{\circ} \mathrm{C}$, however, secondary growth occurs at less frequent locations, and therefore the grains become larger in the earlier stages of annealing. This is indicated in Fig. 9. The $900^{\circ} \mathrm{C} \mathrm{SH}$ sample reaches an earlier saturation at $\sim 30$ minutes with $2.5 \mathrm{~mm}$ average size compared to the $3.5 \mathrm{~mm}$ average size in the $\mathrm{RH}$ annealed sample at $900^{\circ} \mathrm{C}$. It should be noted that the time measurement in the $\mathrm{SH}$ experiments started when the furnace temperature reached $900^{\circ} \mathrm{C}$. Clearly, secondary growth commenced before the temperature reached $900^{\circ} \mathrm{C}$ (see Fig. 4(a)), and therefore secondary recrystallization occurs in more frequent sites and thus yields smaller final grain sizes. Since orientation and size of secondary Goss grains is a function of both annealing temperature and heating rate, it is noted that the smaller grain size and larger orientation spread will have detrimental effect on hysteresis loss, ${ }^{6,7,46)}$ and therefore $900^{\circ} \mathrm{C} \mathrm{RH}$ annealing yields the best texture within short annealing times (i.e. 30 minutes) in the materials investigated.

Finally, in the current material two grain interior features were found with respect to the secondary growth of Goss: crystal defect structures and particle dispersion. A thorough investigation in the as-received sample was conducted by TEM and no evidence of dislocation substructures were found except for a few isolated, residual dislocations as is shown in Fig. 7(a). This is a commonly found feature in many recrystallized metals, ${ }^{35)}$ and therefore are not regarded as evidence to support the theory by Lee, et $a l .{ }^{47)}$ and Ko, et al. ${ }^{48)}$ which correlates the presence of sub-boundaries that enable Goss grains to grow through a solid state wetting mechanism. The particle dispersion in the grain interior of the primary recrystallized grains is found to be similar to that presented by Flowers et al. ${ }^{4)}$ in Si-steels. Although the inhibiting theory is based on the particles at grain boundaries, the grain interior particles are not considered to be less important because when a boundary starts moving and encounters particles in the grain, the boundary must overcome them for continued growth. Therefore, growth becomes favorable if the grains to be consumed contain fewer particles (e.g. by dissolution/coarsening at the annealing temperature) or if the moving boundary acts as the dissolution site when it gets encountered by the particles. ${ }^{27,35)}$ 


\section{Conclusions}

A two-stage cold rolled and primary recrystallized silicon steel was subjected to secondary recrystallization annealing in an air circulating furnace by varying time, temperature and heating rate. The following secondary grain growth phenomena of Goss grains were observed:

- Annealing in a $900-1000^{\circ} \mathrm{C}$ temperature range yields Goss textures through secondary recrystallization. Temperatures above or below this range are not favorable for Goss growth. Isothermal annealing at $900^{\circ} \mathrm{C}$ gives the strongest Goss textures within the experiment variables in this investigation.

- At lower temperature, annealing yields less frequent Goss secondary recrystallization grains and therefore at the completion of the growth process larger Goss grains are formed. Also, at lower annealing temperature sharper Goss texture produces.

- The early growth of secondary grains is more deviated from the ideal Goss orientation because that growth commences at temperatures lower than the critical range i.e. $900-1000^{\circ} \mathrm{C}$. Extended annealing following the completion of secondary recrystallization intensifies the Goss textures by consuming the other oriented grains.

- The island grains in secondary Goss grains become trapped as a result of extended primary growth of certain matrix grains followed by the growth chronology of the growing Goss grains. The distribution of island grains indicates the grain growth mechanism involved during secondary growth of Goss grains. Island grains are randomly oriented and have no specific orientation relationship with the Goss grains containing them.

\section{REFERENCES}

1) M. F. Littmann: J. Magn. Magn. Mater., 26 (1982), 1.

2) M. Matsuo: ISIJ Int., 29 (1989), 809.

3) N. Bernier, E. Leunis, C. Furtado, T. Van De Putte and G. Ban: Micron, 54-55 (2013), 43.

4) J. W. Flowers and S. P. Karas: J. Appl. Phys., 38 (1967), 1085.

5) F. J. Humphreys and M. Hatherly: Recrystallization and Related Annealing Phenomena, Pergamon, Oxford, (2002), 215.

6) G. Abbruzzese and S. Fortunati: J. Appl. Phys., 64 (1988), 5344.

7) P. R. Cha, K. J. Ko, J. T. Park, J. K. Kim and N. M. Hwang: $A d v$. Mater. Res., 26-28 (2007), 65.

8) D. G. Brandon: Acta Metall., 14 (1966), 1479.

9) M. F. Littmann: J. Appl. Phys., 36 (1965), 1225.

10) H. Nakae and K. Tagashira: Trans. Jpn. Inst. Met., 14 (1973), 15.
11) C. Gheorghies and A. Doniga: J. Iron Steel Res. Int., 16 (2009), 78.

12) Y. Ushigami, H. Masui, Y. Okazaki, Y. Suga and N. Takahashi: $J$. Mater. Eng. Perform., 5 (1996), 310.

13) T. Gladman: Scr. Metall. Mater., 27 (1992), 1569.

14) L. A. Barrales Mora, G. Gottstein and L. S. Shvindlerman: Acta Mater., 56 (2008), 5915.

15) J. L. Walter, W. R. Hibbard, H. C. Fiedler, H. E. Grenoble, R. H. Pry and P. G. Frischmann: J. Appl. Phys., 29 (1958), 363.

16) H. C. Fiedler: Metall. Trans. A, 8A (1977), 1307.

17) Y. Ushigami, T. Kubota and N. Takahashi: ISIJ Int., 38 (1998), 553.

18) V. W. Carpenter and J. M. Jackson: US Patent, American Rolling Mill, USA, (1942).

19) E. J. Hilinski: J. Magn. Magn. Mater., 304 (2006), 172.

20) H. Yashiki and T. Fukagawa: J. Magn. Magn. Mater., 160 (1996), 127.

21) Q. Chen, A. Ngan and B. Duggan: Proc. R. Soc. (Lond.), 459 (2003), 1661.

22) D. Dorner, S. Zaefferer and D. Raabe: Acta Mater., 55 (2007), 2519.

23) J. Harase and R. Shimizu: Acta Metall. Mater., 40 (1992), 1101.

24) R. E. Lenhart: J. Appl. Phys., 35 (1964), 861.

25) G. Gottstein, D. A. Molodov, L. S. Shvindlerman, D. J. Srolovitz and M. Winning: Curr. Opin. Solid State Mater. Sci., 5 (2001), 9.

26) B. J. Duggan, M. Sindel, G. D. Köhlhoff and K. Lücke: Acta Metall. Mater., 38 (1990), 103.

27) V. Novikov: Grain Growth and Control of Microstructure and Texture in Polycyrstalline Materials, CRC Press, Boca Raton, (1997), 137.

28) J. Harase and R. Shimizu: Acta Metall. Mater., 38 (1990), 1395.

29) D. Dorner, L. Lahn and S. Zaefferer: Mater. Sci. Forum, 495-497 (2005), 1061.

30) J. E. Burke and D. Turnbull: Prog. Met. Phys., 3 (1952), 220.

31) Y. Inokuti, C. Maeda and Y. Ito: Trans. Iron Steel Inst. Jpn., 27 (1987), 139.

32) Y. Yoshitomi, S. Suzuki, T. Ueda, S. Tsurekawa, H. Nakashima and H. Yoshinaga: Scr. Metall. Mater., 32 (1995), 1067.

33) H. Homma and B. Hutchinson: Acta Mater., 51 (2003), 3795.

34) J. E. May and D. Turnbull: Trans. Metall. Soc. AIME, 212 (1958), 769.

35) N. Afrin, M. Z. Quadir, L. Bassman, J. H. Driver, A. Albou and M. Ferry: Scr. Mater., 64 (2011), 221.

36) Y. Ushigami, T. Nakayama, Y. Suga and N. Takahashi: Mater. Sci. Forum, 204-206 (1996), 605.

37) B. Sokolov, V. Gubernatorov and S. Ketov: Phys. Met. Metallogr., 74 (1992), 193.

38) M. Shinozaki, I. Matoba, T. Kan and T. Gotoh: Trans. Jpn. Inst. Met., 19 (1978), 85.

39) W. Mao, Y. Li, P. Yang and W. Guo: Mater. Sci. Forum, 702-703 (2012), 585 .

40) N. Maazi and R. Penelle: Mater. Sci. Eng. A, 504 (2009), 135.

41) P. A. Beck: Acta Metall., 1 (1953), 230.

42) M. Ferry, M. Z. Quadir, N. Afrin, W. Xu, A. Loeb, B. Soe, C. McMahon, C. George and L. Bassman: 36th Riso Int. Symp. on Materials Science, ed. by S. Faester et al., IOP Publishing, Riso, Denmark, (2015), 13.

$43)$ N. Chen, S. Zaefferer, L. Lahn, K. Gunther and D. Raabe: Mater. Sci. Forum, 408-412 (2002), 949.

$44)$ J. E. May and D. Turnbull: J. Appl. Phys., 30 (1959), S210.

45) M. Z. Quadir, C. S. T. Chang and B. J. Duggan: ISIJ Int., 50 (2010), 264.

46) H. Wriedt and H. Hu: Metall. Trans. A, 7A (1976), 711.

47) K. J. Sixtus: J. Appl. Phys., 6 (1935), 105.

48) K.-J. Ko, P.-R. Cha, D. Srolovitz and N.-M. Hwang: Acta Mater., 57 (2009), 838. 